\title{
Clinical Benefit and Utility of Switching to Aripiprazole Once Monthly in Patients with Antipsychotic Polypharmacy or Long Acting Injectable Antipsychotics for Patients with Schizophrenia in Routine Practice: A Retrospective, Observation Study
}

\author{
Chi-Un Pae ${ }^{1,2,3}$, Changsu Han ${ }^{4}$, Won-Myong Bahk ${ }^{1}$, Soo-Jung Lee ${ }^{1}$, Ashwin A. Patkar ${ }^{2}$, Prakash S. Masand ${ }^{5}$ \\ ${ }^{1}$ Department of Psychiatry, College of Medicine, The Catholic University of Korea, Seoul, Korea, ${ }^{2}$ Department of Psychiatry and Behavioural \\ Sciences, Duke University Medical Center, Durham, NC, USA, ${ }^{3}$ Cell Death Disease Research Center, College of Medicine, The Catholic \\ University of Korea, ${ }^{4}$ Department of Psychiatry, Korea University College of Medicine, Seoul, Korea, ${ }^{5}$ Global Medical Education, New York, \\ NY, USA
}

Objective: In a number of controlled clinical trials and naturalistic studies, aripiprazole once monthly (AOM) has been found to be effective and safe as acute and maintenance treatment options for schizophrenia. However, such clinical data have been presented in selected patient population (i.e., antipsychotic monotherapy, etc.), in particular, clinical information on switching to AOM from antipsychotic polypharmacy and/or other long acting injectable antipsychotics (LAIs) has been scarce till today.

Methods: The study period was from the first switching day to AOM up to 12 months in patients with antipsychotic polypharmacy (APpoly)/LAls (baseline, month 3, month 6, and month 12). Available demographics and clinical information were retrieved from electronic medical records (EMRs). Available scores of Global Assessment of Functioning (GAF), Clinical Global Impression-Clinical Benefit (CGI-CB), CGl-severity, Visual Analog Scale on Satisfaction-Patient/Health Professional (VAS-P/HP), and the Positive and Negative Syndrome Scale-Insigh (PANSS-I) scores were also taken from EMR. Proportional change of functional impairment before and after AOM was also captured.

Results: Data of 18 patients were available. Most commonly used combined APs before AOM were aripiprazole, blonanserin, quetiapine, and risperidone. At least $2 \mathrm{APs}(\mathrm{n}=2.4)$ were combined before AOM. Scores of GAF $(10.7 \%$ increase), CGI-CB (46.2\% decrease), VAS-P (47.8\% increase), VAS-HP (40.8\% increase), and PANSS-I (27.9\% increase) (all $p=0.001$ ) were significantly improved from baseline to month 12, respectively. Approximately $59 \%$ of patients improved individual functioning with different level (i.e., employment, back to school, etc.) after AOM treatment at month 12 .

Conclusion: The present study have clearly shown the clinical benefit and utility of switching to AOM for treatment of patients with APpoly/LAls in routine practice. Subsequent, adequately-powered, well-controlled clinical trials may be necessary to confirm our findings in near future.

KEY WORDS: Aripiprazole once monthly; Long-acting injectable antipsychotics; Polypharmacy; Clinical utility; Schizophrenia; Benefit.

\section{INTRODUCTION}

Aripiprazole once monthly (AOM) has been approved

Received: October 25, 2019 / Revised: December 9, 2019

Accepted: December 18, 2019

Address for correspondence: Chi-Un Pae

Department of Psychiatry, Bucheon St. Mary's Hospital, College

of Medicine, The Catholic University of Korea, 327 Sosa-ro,

Wonmi-gu, Bucheon 14647, Korea

E-mail: pae@catholic.ac.kr

ORCID: https://orcid.org/0000-0003-1632-4248
2013 for treatment of schizophrenia [1]. It is one of promising long acting injectable antipsychotics which have many advantages for management of patients with schizophrenia proven through a number of well-designed controlled (RCTs) [2,3] and naturalistic clinical trials as well as meta-analysis and claim data study, particularly those who have compliance/adherence issues in clinical practice [4-14]. According to such pivotal RCTs [4,5], it has superiority over placebo in lowering rehospitalization as

(c) This is an Open-Access article distributed under the terms of the Creative Commons Attribution Non-Commercial License (http://creativecommons.org/licenses/by-nc/4.0) which permits unrestricted non-commercial use, distribution, and reproduction in any medium, provided the original work is properly cited. 
well as shown clear efficacy and safety as acute, maintenance, and long-term treatment options for schizophrenia [4-10].

The ultimate goal of treatment for patients with schizophrenia has shifted from remission and response to biological treatment to recovery of personal, social, and occupational functional capacity as well as reintegration into public and social community [15]. Indeed a lower proportion of patients regain proper personal and social functioning, while $30 \%$ to $70 \%$ of patients reach symptomatic remission after proper antipsychotic treatment [16]. Therefore, the functional deficits in schizophrenia, independent of psychopathology, has been considered one of the most urgent, important and crucial assessments for the efficacy of antipsychotic therapy [17]. In both pivotal trials [4,5] and acute study [8], patients' functioning measured by Personal and social Performance sale (PSP) was also more significantly improved in AOM group than in placebo group. In addition, a recent comparative trial of AOM vs. paliperidone palmitate (PP), AOM has shown significantly greater improvement in functioning over PP by difference of 4.7 point measured by Heinrichs-Carpenter Quality-of-Life Scale (QLS), in which such difference was more pronounced in patients $\leq 35$ years on QLS (10.7 point difference) [18]. Moreover, such effect was also continued with small but further improvements in a subsequent 28 weeks extension study [19]. Additionally, in a recent naturalistic study investigating the effectiveness of AOM in regular clinical settings, AOM has also demonstrated its effects on functioning by $25 \%$ increase measured by Global Assessment of Functioning score (GAF) as well as on psychopathology change ( $33 \%$ improvement) measured by Clinical Global Impression-Severity (CGI-S) score [20]. These favourable effects of AOM on functioning measured by PSP score was more prominent in patients $\leq 35$ years in a recent post-hoc analysis [17] of an acute phase trial [8] and 52-week extension study [21]. For instance, in an acute phase trial [8], the improvement of functioning measured by PSP scores was statistically favourable to AOM over placebo in patients $\leq 35$ years by a difference of 11.1 points, while it was numerically favourable to AOM over placebo in patients $>35$ tears by a difference of 5.9 points. Furthermore, improvements in both age groups met criteria for a minimally important clinical difference $(7-10$ points) [18]. Despite of no statistical significance, small consistent numerical improvements of the PSP score (approximately 1 point) was also demonstrated in 52-week extension study [21].

Given that previous findings, AOM has shown its clear and promising effects on psychopathology and functioning in a number of RCTs and naturalistic studies. However, such clinical efficacy including positive role on functioning of AOM have been mainly demonstrated in selected patient population by rigorous inclusion criteria (i.e., entry criteria for psychopathology, comorbidity, concomitant drug, etc.) not reflecting real world patient population. Additionally strict switching and stabilization period to oral aripiprazole (monotherapy) from current different antipsychotics and switching schedule to AOM were very conservative due to their controlled study design $[4,5,8,9,20]$. Furthermore, switching from antipsychotic polypharmacy (APpoly) to AOM has not been demonstrated yet, as is in switching to different long acting injectable antipsychotics (LAIAs), paliperidone palmitate [22]. In fact APpoly is common in real world practice ranging from $4 \%$ to $70 \%$ depending methodologies [23], such trend was also replicated in many claim-data and cohort studies $[24,25]$. Thus accumulation of further clinical information about switching to AOM from APpoly/LAl should be necessary to safe use of AOM and proper evaluation of its effects for such patient population on functioning and other relevant areas in routine practice.

In fact we previously reported substantial usefulness of switching to AOM in patients with APpoly/LAI demonstrating its clinical effectiveness and tolerability by measurements of psychopathology scales and adverse events (AEs) [26]. Therefore, the present study aimed to test the clinical utility and benefit of switching to AOM for in patients who were on APpoly/LAls in routine practice, in terms of functioning, satisfaction, and acceptability utilizing several scales which are commonly applied in clinical practice.

\section{METHODS}

The present study was a non-interventional, retrospective, 12-month, observational study. Various clinical assessments using scales were done during the regular clinic visits which were the part of routine care (baseline, first AOM injection, month 3, month 6 , and month 12 ).

Data were collected from January 2017 to August 2019 based on patients' electronic medical records (EMR). Eighteen 
patients EMRs were selected based on the following criteria: 1) any type of the Diagnostic and Statistical Manual of Mental Disorders 5th edition schizophrenia; 2) patients who were on 2 or more oral APs (OAPs) or different LAls ( $\geq 3$ months) before baseline; 3) patients should have at least 3 shots of AOM after baseline; 4) patients should have stable diagnosis of schizophrenia at least for more than 2 year, and 5) otherwise, no exclusion criteria were applied to reflect naturalistic treatment setting.

Age, sex, education level, family history, comorbidity, duration of illness, number of admission, number of treatment failure, and diverse pharmacological data were collected. Available scores of GAF, Clinical Global ImpressionClinical Benefit (CGI-CB), CGI-S, Visual Analog Scale on Satisfaction-Patient/Health Professional (VAS-P/HP), and the Positive and Negative Syndrome Scale (PANSS)-Insight scores were retrieved at each visit. The PANSS-total, -positive, -negative, and -general scores as well as CGI-S score were also collected at each visit. All AEs reported in EMR were also presented throughout the observation period.

All patients who had at least 3 shots of AOM were included for the outcome and tolerability analyses as priori definition of inclusion. Last observation carried forward was applied to impute missing or short follow-up data due to study period. Descriptive statistics were performed using the mean \pm standard deviation and frequency $(\%)$ for continuous and categorical variables for basic demographics and clinical information. To compare changes in psychopathology and other clinical outcomes from baseline to month 12 , nonparametric tests were done where appropriate. For exploratory purposes, a one-way general linear model was also conducted to see the trend of time-effects on psychopathology and other clinical outcomes changes. Group differences by follow-up periods (up to 12 months vs. $<12$ months; $\geq 6$ months vs. $<6$ months) and illness course (early phase: $\leq 5$ years vs. late phase: $>5$ years) in psychopathology and other clinical outcomes were also compared by nonparametric tests where appropriate. All statistical tests were two-sided and a $p$ value of $\leq 0.05$ was considered significant.

The study was approved by the institutional review board (IRB) at Bucheon St. Mary's Hospital and was conducted in compliance with the Declaration of Helsinki (IRB no. HC19RESE0089).

\section{REUSLTS}

As we reported detailed demographic, clinical and pharmacological data in our previous study (submitted), only brief and essential results on them are presented here. A total of 18 patients were enrolled and took at least 3 shots of AOM during the study. Among 18 patients, 12 $(66.7 \%)$ completed 12 months full observation. Male proportion was $50 \%$ and mean age was 39.6. Duration of illness, number of admission, and previous treatment antipsychotic failure were 7.9 years, 1.2 , and 2.2. Only one patient has family history of psychiatric disorder. Six patients (33.3\%) had comorbid medical conditions and approximate half of patients $(n=8)$ had graduated or attended college. Details of major psychometric scales are represented in Table 1.

The most frequently combined OAPs immediate before AOM use were, aripiprazole, blonanserin, quetiapine, and risperidone, while paliperidone palmitate was the only LAI. The mean numbers of OAPs immediate before AOM were 2.4. The combined mood stabilizers and antiparkinsonian drugs immediate before AOM were used in $11.1 \%$ and $44.4 \%$ of patients. The mean dose of AOM and OARP at baseline were $344.4 \mathrm{mg}$ and $7.2 \mathrm{mg} / \mathrm{d}$, respectively.

\section{PANSS and CGI Scores}

The PANSS total score was significantly decreased by $13.6 \%$ from baseline to month $12(-9.8, p=0.001)$. When it comes to subscores of the PANSS, the PANSS positive, negative, and general scores all significantly decreased by $10.7 \%(p=0.015), 21.3 \%(p=0.001)$, and

Table 1. Baseline demographics of the sample

\begin{tabular}{cc}
\hline Variable & Mean \pm standard deviation \\
\hline PANSS total score & $71.9 \pm 9.2$ \\
PANSS positive & $19.9 \pm 3.6$ \\
PANSS negative & $24.0 \pm 6.1$ \\
PANSS general & $28.9 \pm 7.4$ \\
CGI-S & $3.4 \pm 0.7$ \\
CGI-CB & $7.8 \pm 1.5$ \\
GAF & $49.7 \pm 12.0$ \\
VAS-P & $4.6 \pm 1.1$ \\
VAS-HP & $4.9 \pm 1.5$ \\
PANSS-Insight & $4.3 \pm 1.1$ \\
\hline
\end{tabular}

PANSS, The Positive and Negative Syndrome Scale; CGI-S, Clinical Global Impression-Severity; CGI-CB, CGI-Clinical Benefit; GAF, Global Assessment of Functioning; VAS-P/HP, Visual Analog Scale for satisfaction-Patient/Health professional. 
$13.6 \%(p=0.001)$, respectively from baseline to month 12. The CGI-S score was also significantly decreased by $8.8 \%$ from baseline to month $12(-0.3, p=0.008)$.

When compared the two groups, 12 months completers showed significantly greater improvements in PANSS total and negative scores than non-completer, while there were no group differences in scores of PANSS positive, general and CGI-S (Supplementary Table 1; available online). In addition, when subdividing patients 6 months completers and non-completer, there were no group differences in all psychopathology scores (data not shown).

\section{GAF Score}

The GAF score was significantly increased by $10.9 \%$ from baseline to month 12 (5.4, $p=0.001$, Fig. 1). Furthermore, the linear increase trend was substantial in change of GAF score during the study period ( $p=0.005)$. When compared the two groups, 12 months completers showed significantly greater improvements in GAF score than non-completer ( $p=0.007$, Supplementary Fig. 1; available online). In addition, when subdividing patients 6 months completers and non-completer, there were no group differences in change of GAF score (Supplementary Fig. 2; available online).

\section{CGI-CB Score}

The CGI-CB score was significantly decreased by $46.2 \%$ from baseline to month $12(-3.6, p=0.001$, Fig. 2). Furthermore, the linear decrease trend was substantial

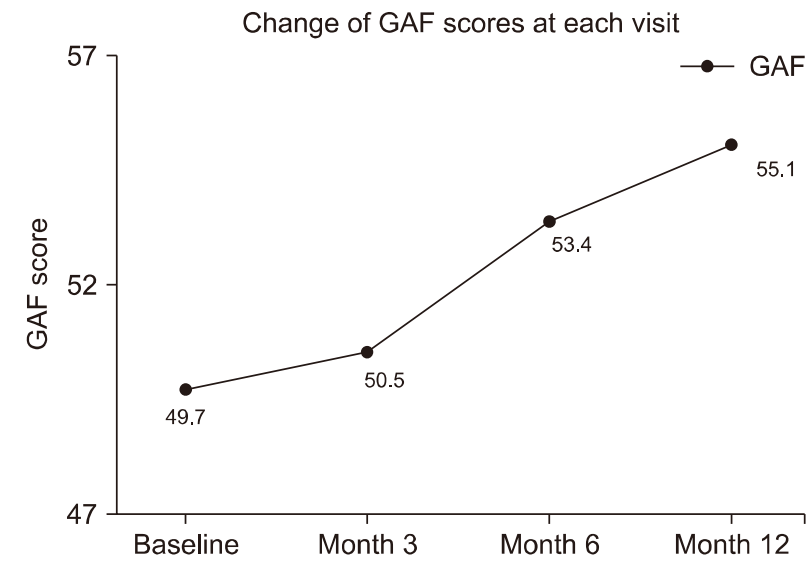

Fig. 1. The change of Global Assessment of Functioning (GAF) score during the study. $p=0.109,0.007,0.005$, and 0.001 between baseline to month 3, month 3 to month 6 , month 6 to month 12 , and baseline to month 12, respectively, Wilcoxon Signed Rank test; Time effect, $\mathrm{df}=3, \mathrm{~F}=6.506, p=0.005$, one way GLM. in change of CGI-CB score during the study period ( $p=$ 0.001). When compared the two groups, 12 months completers showed significantly greater improvements in CGI-CB score than non-completer ( $p=0.007$, Supplementary Fig. 1; available online). In addition, when subdividing patients 6 months completers and non-completer, there were no group differences in in change of CGI-CB score (Supplementary Fig. 2; available online).

\section{VAS-P Score}

The VAS-P score was significantly increased by $47.8 \%$ from baseline to month $12(2.2, p=0.001$, Fig. 3).

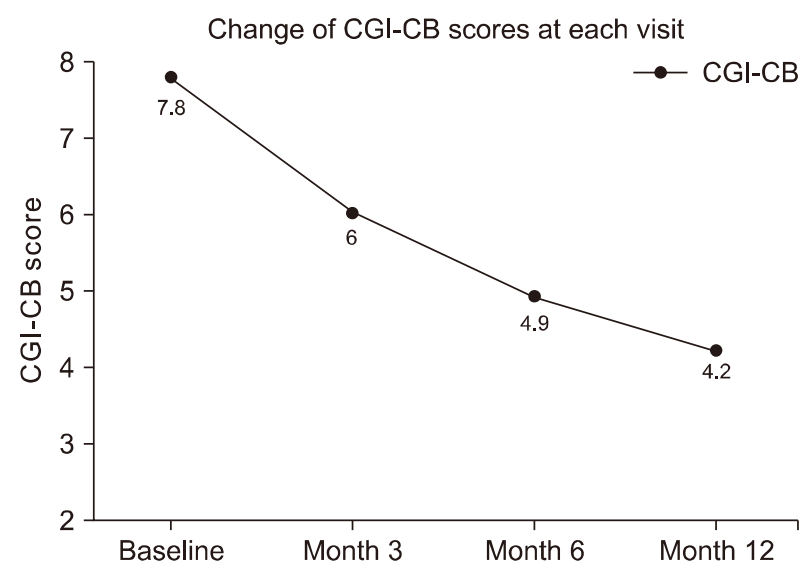

Fig. 2. The change of Clinical Global Impression-Clinical Benefit (CGI-CB) score during the study. $p=0.001,0.003,0.016$, and 0.001 between baseline to month 3, month 3 to month 6 , month 6 to month 12 , and baseline to month 12, respectively, Wilcoxon Signed Rank test; Time effect, $\mathrm{df}=3, \mathrm{~F}=10.682, p=0.001$, one way GLM.

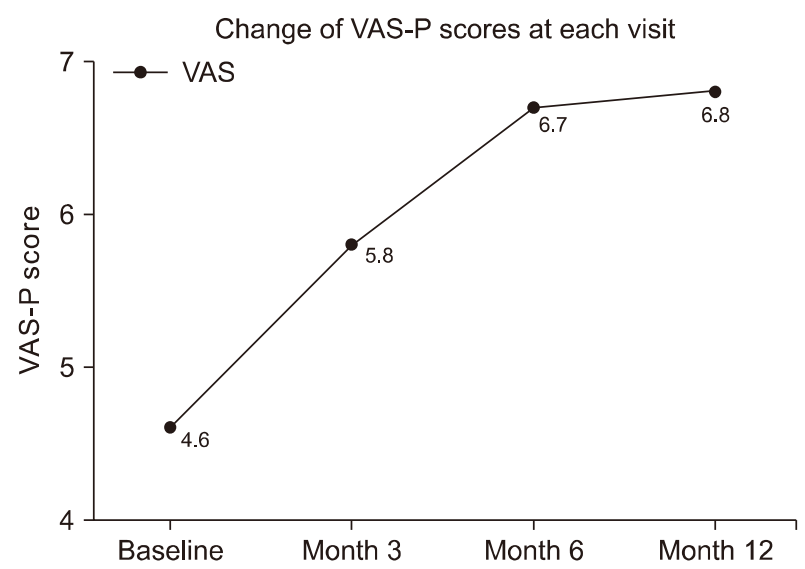

Fig. 3. The change of Visual Analog Scale-Patient (VAS-P) score during the study. $p=0.001,0.001,0.480$, and 0.001 between baseline to month 3, month 3 to month 6 , month 6 to month 12, and baseline to month 12, respectively, Wilcoxon Signed Rank test; Time effect, $\mathrm{df}=3, \mathrm{~F}=16.162, p=0.000$, one way GLM. 


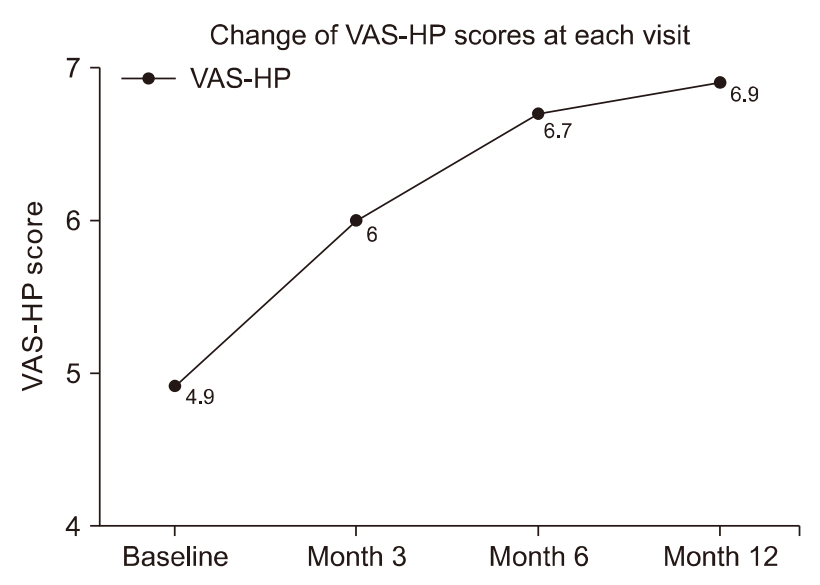

Fig. 4. The change of Visual Analog Scale-Health Professional (VAS-HP) score during the study. $p=0.001,0.006,0.279$, and 0.001 between baseline to month 3 , month 3 to month 6 , month 6 to month 12 , and baseline to month 12, respectively, Wilcoxon Signed Rank test; Time effect, $\mathrm{df}=3, \mathrm{~F}=9.323, p=0.001$, one way GLM.

Furthermore, the linear increase trend was substantial in change of VAS-P score during the study period $(p=$ 0.000 ). When compared the two groups, 12 months completers showed significantly greater improvements in VAS-P score than non-completer $(p=0.018$, Supplementary Fig. 1; available online). In addition, when subdividing patients 6 months completers and non-completer, there were no group differences in in change of VAS-P score (Supplementary Fig. 2; available online).

\section{VAS-HP Score}

The VAS-HP score was significantly increased by $10.9 \%$ from baseline to month 12 (2.0, $p=0.001$, Fig. 4). Furthermore, the linear increase trend was substantial in change of VAS-HP score during the study period ( $p=$ 0.001). When compared the two groups, 12 months completers showed significantly greater improvements in VAS-HP score than non-completer ( $p=0.010$, Supplementary Fig. 1; available online). In addition, when subdividing patients 6 months completers and non-completer, there were no group differences in in change of VAS-HP score (Supplementary Fig. 2; available online).

\section{PANSS-Insight Score}

The PANSS-Insight score was significantly decreased by $27.9 \%$ from baseline to month $12(-1.2, p=0.001$, Fig. 5). Furthermore, the linear decrease trend was substantial in change of PANSS-Insight score during the study period $(p=0.000)$. When compared the two groups, there

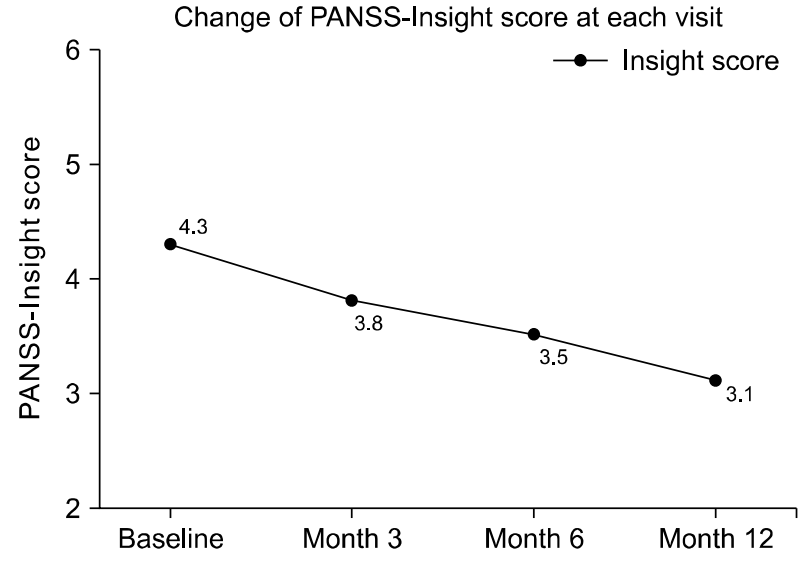

Fig. 5. The change of the Positive and Negative Syndrome Scale-Insight (PANSS-Insight) score during the study. $p=0.003$, $0.025,0.008$, and 0.001 between baseline to month 3 , month 3 to month 6, month 6 to month 12, and baseline to month 12, respectively, Wilcoxon Signed Rank test; Time effect, $\mathrm{df}=3, \mathrm{~F}=$ $11.719, p=0.000$, one way GLM.

were no group differences in PANSS-Insight score between 12 months completers and non-completer (Supplementary Fig. 1; available online). In addition, when subdividing patients 6 months completers and non-completer, there were no group differences in in change of PANSS-Insight score (Supplementary Fig. 2; available online).

\section{Proportion of patients of functional improvement from baseline}

Functional impairment included failure to work, enjoy social program/activity, or regularly attend employment rehabilitation program in routine life; only one patient had regular job at baseline, while 11 patients were found to have substantial improvement in functioning with different level at month 12 (for instance, among 11 patients, 4 have regular job, 2 have part-time job, 4 participate in regular social program provided by local government and community resources, and 1 attends employment rehabilitation program ( $p=0.002$, McNemar Test, Fig. 6).

\section{Correlations between rating scales}

When we analysed the correlation between changes in PNASS positive, negative, and general scores, GAF score, VAS-P score, PANSS-Insight score from baseline to month 12 after controlling age, sex, duration of illness, treatment failure number, baseline PANSS total score, and admission number, there were significant correlations between 
PANSS negative score. The details of results are presented in Table 2. Briefly, the change of GAF score was significantly correlated with PANSS negative, CGI-CB, and VAS-P/HP scores. In addition, the PANSS-Insight score was significantly correlated with PANSS negative score.

Proportion of functional impairment before and after AOM

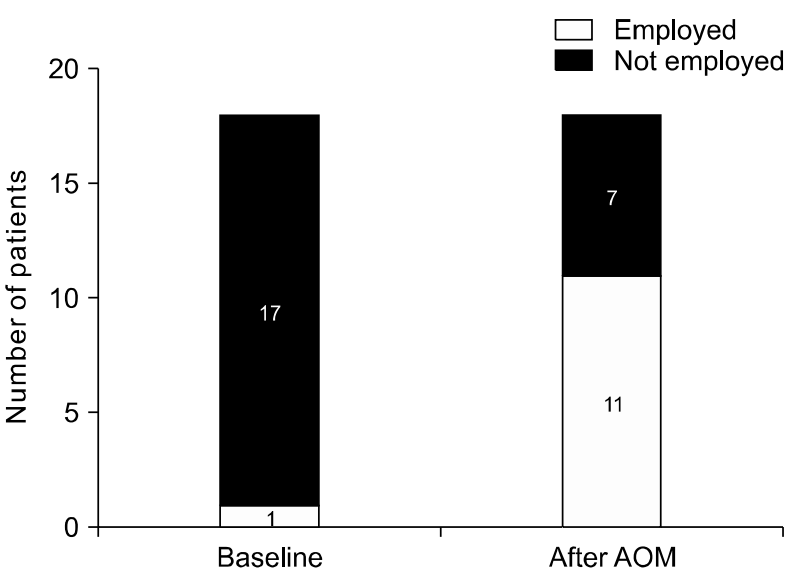

Fig. 6. The change of proportion of patients of improvement in functional impairment during the study. Functional impairment means that patients did not have work, enjoy social program/activity, or regularly attend employment rehabilitation program in routine life (for instance, among 11 patients, 4 have regular job, 2 have part-time job, 4 participate in regular social program provided by local government and community resources, and 1 attends employment rehabilitation program; Exact significance (two-tailed) $=0.002$, McNemar Test.

AOM, aripiprazole once monthly.

\section{Group differences in rating scales by age}

There have been no statistical differences between patients aged $\leq 35$ years and $>35$ years in changes of all rating scale scores from baseline to month 12 in the present study. The proportion of patients who regained functional capacity at month 12 was not also significantly different by patients aged $\leq 35$ years and $>35$ years (data not shown).

\section{DISCUSSION}

Overall switching to AOM in patients with APpoly/LAI (SWAOM) has clearly shown clinical utility and benefit assessed by multiple rating scales including GAF, CGI-CB, VAS-P/HP, and PANSS-Insight scores in the present study. In addition, 12 months completers have demonstrated significantly more improvement in GAF, CGI-CB, and VAS-P/HP scores excluding PANSS-Insight score than non-completer, indicating that clinical utility and benefit of SWAOM may be more pronounced by longer maintenance treatment than by short-term treatment as expected by its formulation characteristics and purpose of the use of AOM. Furthermore, more than half of patients (10/17) achieved substantial functional improvement with different level by returning to work, participating social activities, or back to school after treatment of AOM at month 12. Such improvement in functioning level also clearly support the usefulness of SWAOM in daily prac-

Table 2. Correlation between changes in PANSS positive, negative, and general scores, CGI-CB score, GAF score, VAS-P score, and PANSS-Insight score from baseline to month 12

\begin{tabular}{lrccccccc}
\hline \multirow{2}{*}{ Parameters } & PANSS & PANSS & PANSS & \multirow{2}{*}{ CGI-CB } & GAF & VAS-P & PANSS-Insight & VAS-HP \\
& positive & negative & general & & & & \\
\hline \multirow{2}{*}{ CGI-CB } & 0.405 & 0.383 & 0.633 & 1.000 & -0.702 & -0.617 & 0.176 & -0.658 \\
& 0.191 & 0.219 & $0.027^{\mathrm{a}}$ & - & $0.011^{\mathrm{a}}$ & $0.033^{\mathrm{a}}$ & 0.584 & $0.020^{\mathrm{a}}$ \\
GAF & -0.478 & -0.647 & -0.547 & -0.702 & 1.000 & 0.674 & -0.208 & 0.767 \\
& 0.116 & $0.023^{\mathrm{a}}$ & 0.065 & $0.011^{\mathrm{a}}$ & - & $0.016^{\mathrm{a}}$ & 0.517 & $0.004^{\mathrm{a}}$ \\
VAS-P & -0.416 & -0.712 & -0.778 & -0.617 & 0.674 & 1.000 & -0.510 & 0.762 \\
& 0.179 & $0.009^{\mathrm{a}}$ & $0.003^{\mathrm{a}}$ & $0.033^{\mathrm{a}}$ & $0.016^{\mathrm{a}}$ & - & 0.091 & $0.004^{\mathrm{a}}$ \\
PANSS-Insight & 0.341 & 0.612 & 0.412 & 0.176 & -0.208 & -0.510 & 1.000 & -0.181 \\
\multirow{2}{*}{ VAS-HP } & 0.279 & $0.034^{\mathrm{a}}$ & 0.183 & 0.584 & 0.517 & 0.091 & - & 0.574 \\
& -0.314 & -0.400 & -0.573 & -0.658 & 0.767 & 0.762 & -0.181 & 1.000 \\
& 0.321 & 0.198 & 0.052 & $0.020^{\mathrm{a}}$ & $0.004^{\mathrm{a}}$ & $0.004^{\mathrm{a}}$ & 0.574 & - \\
\hline
\end{tabular}

Upper column indicates correlation and lower column indicates significance. Partial correlation after controlling age, sex, admission number, duration of illness, treatment failure number, and baseline PANSS total score.

PANSS, The Positive and Negative Syndrome Scale; CGI-CB, CGI-Clinical Benefit; GAF, Global Assessment of Functioning; VAS-P/HP, Visual Analog Scale for satisfaction-Patient/Health professional; -, not available.

${ }^{\mathrm{a}}$ Significant values. 
tice and is in line with previous study results from acute and long-term RCTs and large naturalistic studies utilized switching to AOM from antipsychotic monotherapy $[4,5,8,19,20]$.

In our study, the change of GAF score was 5.4 points $(10.7 \%)$ increase from 49.7 at baseline to 55.1 at month 12 , which indicates a 1-category improvement from "serious symptoms or any serious impairment in social, occupational, or school functioning" to "moderate symptoms or moderate difficulty in social, occupational, or school functioning". Such difference was smaller than that from previous study demonstrating 2-category improvement, 12 points increase (24.5\%) from 49 to 61 in a recent naturalistic study conducted in Canada [20]. Such difference might be originated from different clinical characteristics of subjects (polypharmacy, duration of illness, age, sex distribution, and range of GAF, etc.), study design (heterogeneity of subjects, single-centre vs. multi-centre, etc.), and sample size, etc. However, based on classification of GAF by American Psychiatric Association, GAF score of 55 is the top of moderate impairment level and $\geq$ 60 points are the indicator of good functioning [27]. Additionally, $<50$ points were found to significantly increase a risk of relapse but $51-60$ points of GAF were not different in the risk of developing relapse, compared to $\geq$ 60 points [27]. Interestingly $\geq 50$ points was also considered of remission criteria [28]. It is also interesting that GAF improvement was $14 \%$ (6.8 points from 39.4 to 46.2 ) in a previous 6-month oral aripiprazole study [29], which is similar to our findings. Of note, 8 points $(16.1 \%) \mathrm{im}$ provement of GAF was found in 12 month completers in our study, indicating 1.5 folds increase than whole sample analysis. Given that previous study findings, 12 months of treatment, mean change of approximately 7.0 in GAF from baseline, 20\% power, and a coefficient of variation of 1 , the proper sample size should be at least 189 patients, indicating that 5.4 increase of GAF score is remarkable upon reflection of our small sample size [20]. Significant correlations in the GAF and CGI-S or PANSS scores, both cross-sectionally and longitudinally was found, particularly, between GAF and CGI-S scores, which was also observed in our study [30]. Furthermore, a previous study roughly assumed that a 0.8-point decrease of CGI-S may be translated into 10-point increase (one category) in the GAF, which is equivalent to a $33 \%$ decrease in the PANSS [30]. This is well-reflected in our present study since the increase of GAF, decrease of CGI-S, and decrease of PANSS scores were 5.4-point, 0.3-point, and $13.6 \%$, respectively. Finally, A recent study [31] found, using equipercentile linking, that a CGI-S of 3 and 4 corresponded to a GAF of $52-54$ and $48-51$, respectively, which is more closed to our findings rather than Mustafa and colleagues study [20].

The CGI-CB has been found to be useful in the assessment of acceptability of treatments in routine practice since it evaluates both aspects of efficacy and AEs within very short time [32-34]. A score of 1, indicating greatest benefit (effectiveness) with least burden (AEs) from treatment, to 10 , indicating the least benefit with the greatest burden. In our CGI-CB score significantly decreased by $46.2 \%$ from baseline (7.8 to 4.2 ) at month $12, \leq 4$ in CGI-CB is usually considered outweighing therapeutic effects over AEs [33,35]. The safety and tolerability as well as profound efficacy of AOM has been well-established in a number of previous controlled and naturalistic studies which are in line with the findings from the present study.

Insight is considered one of most important patient factors influencing treatment outcome since it involves multidimensional construct that comprises aspects such as awareness of the disorder and recognition of the need for treatment [36]. In the present study, the PANSS-Insight score significantly decreased by $27.9 \%$ from baseline at month 12 , indeed it was 4.3 at baseline while 3.1 at month 12. The change of PANSS-Insight score from 4 to 3 indicates improvement from moderate to mild impairment [37], indeed the PANSS-Insight score of $<4$ was found to have significant moderating effects on subjective life satisfaction, multiple domain of symptoms, medication compliance, and daily functioning [28,37].

In the present study, patients' and health professionals' satisfaction measured by VAS-P/HP were significantly increased by $47.8 \%$ and $40.8 \%$, respectively. These improvement were also highly correlated with acceptability and functioning as well as PANSS-negative score in the present study. The patient-centered approach and therapeutic alliance are considered key to achieving optimal outcomes in patients with schizophrenia, in particular, patients' satisfaction is crucially associated with long-term outcomes in patients with schizophrenia [38]. In fact, many studies [39-41] have shown clear improvement of patients' satisfaction after switching to LAI from oral OAPs, interestingly, such patients' satisfaction maintained 
even in long-term treatment with AOM [41], which also support the present finding. In addition, multidimensional superiority of AOM over PP has been clearly found and maintained for long-term treatment period $[19,40]$.

Finally, our study has many drawbacks for generalization. The sample size was not adequately large to detect a large effect size upon reflection of many rating scales used in the study, however, we have to consider that SWAOM has not been studied yet. All the patients were recruited in one university-based teaching hospital. Naturalistic and retrospective design could influence outcome results due to recall bias and lack of control group. Finally, we have investigated multi-dimensional outcomes including function, insight, acceptability, and satisfaction which may be involved in occurrence of multiple comparisons. However, LAIAs are well-known to increase treatment compliance and adherence by which patients could maintain medication effects due to retaining therapeutic drug level based on the characteristics of such formulation for a certain period [42]. In addition, LAIAs have also positive potential to improve insight level according to previous researches. In particular prominent effects of AOM on function has been consistently replicated even in direct comparison studies with competitive LAIA $[17,19,20]$. Furthermore, functioning, insight, and subjective satisfaction are also interactive and well-correlated each other [37]. Thus, the present study tried to investigate multi-dimensional outcomes after treatment of AOM to obtain more real world information.

In conclusion, the present study indicates that AOM may be also clinically beneficial and useful treatment option in terms of functioning, satisfaction, and acceptability for patients with currently being treated by antipsychotic polypharmacy in routine practice without worsening of psychopathology or causing serious AEs. Adequatelypowered and well-controlled clinical trials would provide more generalizable information and support the present findings in near future.

\section{- Conflicts of Interest}

No potential conflict of interest relevant to this article was reported.

\section{- Author Contributions}

Conceptualization: Chi-Un Pae, Changsu Han. Protocol evelopment: Chi-Un Pae. Draft writing: Chi-Un Pae.
Intellectual comments and critics on the content: SooJung Lee, Won-Myong Bahk, Ashwin A. Patkar, and Prakash S. Masand. Data acquisition: Chi-Un Pae. Data analysis: Chi-Un Pae, Ashwin A. Patkar, and Prakash S. Masand.

\section{ORCID}

Chi-Un Pae https://orcid.org/0000-0003-1632-4248

Changsu Han https://orcid.org/0000-0002-4021-8907

Won-Myong Bahk https://orcid.org/0000-0002-0156-2510

Soo-Jung Lee https://orcid.org/0000-0002-1299-5266

Ashwin A. Patkar https://orcid.org/0000-0003-0484-1301

Prakash S. Masand https://orcid.org/0000-0003-1973-9449

\section{REFERENCES}

1. Otsuka Pharmaceutical Co., Ltd. The manual of Abilify Maintena Kit. Tokyo:Otsuka Pharmaceutical Co., Ltd.;2013.

2. Pae CU, Wang SM, Han C, Bahk WM, Lee SJ, Patkar AA, et al. Comparison between long-acting injectable aripiprazole versus paliperidone palmitate in the treatment of schizophrenia: systematic review and indirect treatment comparison. Int Clin Psychopharmacol 2017;32:235-248.

3. Park MH, Han C, Pae CU, Lee SJ, Patkar AA, Masand PS, et al. Aripiprazole treatment for patients with schizophrenia: from acute treatment to maintenance treatment. Expert Rev Neurother 2011;11:1541-1552.

4. Fleischhacker WW, Sanchez R, Perry PP, Jin N, Peters-Strickland $\mathrm{T}$, Johnson BR, et al. Aripiprazole once-monthly for treatment of schizophrenia: double-blind, randomised, non-inferiority study. Br J Psychiatry 2014;205:135-144.

5. Kane JM, Sanchez R, Perry PP, Jin N, Johnson BR, Forbes RA, et al. Aripiprazole intramuscular depot as maintenance treatment in patients with schizophrenia: a 52-week, multicenter, randomized, double-blind, placebo-controlled study. J Clin Psychiatry 2012;73:617-624.

6. Ishigooka J, Nakamura J, Fujii Y, Iwata N, Kishimoto T, Iyo M, et al. Efficacy and safety of aripiprazole once-monthly in Asian patients with schizophrenia: a multicenter, randomized, double-blind, non-inferiority study versus oral aripiprazole. Schizophr Res 2015;161:421-428.

7. Potkin SG, Raoufinia A, Mallikaarjun S, Bricmont P, PetersStrickland T, Kasper W, et al. Safety and tolerability of once monthly aripiprazole treatment initiation in adults with schizophrenia stabilized on selected atypical oral antipsychotics other than aripiprazole. Curr Med Res Opin 2013;29:12411251.

8. Kane JM, Peters-Strickland T, Baker RA, Hertel P, Eramo A, Jin $\mathrm{N}$, et al. Aripiprazole once-monthly in the acute treatment of schizophrenia: findings from a 12-week, randomized, double-blind, placebo-controlled study. J Clin Psychiatry 2014; 75:1254-1260. 
9. Fleischhacker WW, Sanchez R, Johnson B, Jin N, Forbes RA, McQuade $\mathrm{R}$, et al. Long-term safety and tolerability of aripiprazole once-monthly in maintenance treatment of patients with schizophrenia. Int Clin Psychopharmacol 2013;28:171 176.

10. Kane JM, Sanchez R, Zhao J, Duca AR, Johnson BR, McQuade $\mathrm{RD}$, et al. Hospitalisation rates in patients switched from oral anti-psychotics to aripiprazole once-monthly for the management of schizophrenia. J Med Econ 2013;16:917-925.

11. Cameron C, Zummo J, Desai D, Drake C, Hutton B, Kotb A, et al. Efficacy and safety of aripiprazole lauroxil once-monthly versus aripiprazole once-monthly long-acting injectable formulations in patients with acute symptoms of schizophrenia: an indirect comparison of two double-blind placebo-controlled studies. Curr Med Res Opin 2018;34:725-733.

12. Schöttle D, Janetzky W, Luedecke D, Beck E, Correll CU, Wiedemann K. Effectiveness of aripiprazole once-monthly in schizophrenia patients pretreated with oral aripiprazole: a 6-month, real-life non-interventional study. BMC Psychiatry 2018; 18:365.

13. Yan T, Greene M, Chang E, Hartry A, Touya M, Broder MS. Medication adherence and discontinuation of aripiprazole once-monthly $400 \mathrm{mg}$ (AOM 400) versus oral antipsychotics in patients with schizophrenia or bipolar I disorder: a real-world study using US claims data. Adv Ther 2018;35: 1612-1625.

14. Wang SM, Han C, Lee SJ, Patkar AA, Masand PS, Pae CU. Schizophrenia relapse and the clinical usefulness of oncemonthly aripiprazole depot injection. Neuropsychiatr Dis Treat 2014;10:1605-1611.

15. Juckel G, de Bartolomeis A, Gorwood P, Mosolov S, Pani L, Rossi A, et al. Towards a framework for treatment effectiveness in schizophrenia. Neuropsychiatr Dis Treat 2014;10: 1867-1878.

16. Bobes J, Ciudad A, Alvarez E, San L, Polavieja P, Gilaberte I. Recovery from schizophrenia: results from a 1-year follow-up observational study of patients in symptomatic remission. Schizophr Res 2009; 115:58-66.

17. Peters-Strickland T, Baker RA, Such P, Zhang P, Madera JJ. The effect of aripiprazole once-monthly on personal and social functioning: post hoc analyses of acute and long-term studies. Neuropsychiatr Dis Treat 2019;15:1659-1669.

18. Naber D, Hansen K, Forray C, Baker RA, Sapin C, Beillat M, et al. Qualify: a randomized head-to-head study of aripiprazole once-monthly and paliperidone palmitate in the treatment of schizophrenia. Schizophr Res 2015;168:498-504.

19. Naber D, Baker RA, Eramo A, Forray C, Hansen K, Sapin C, et al. Long-term effectiveness of aripiprazole once-monthly for schizophrenia is maintained in the QUALIFY extension study. Schizophr Res 2018;192:205-210.

20. Mustafa S, Bougie J, Miguelez M, Clerzius G, Rampakakis E, Proulx J, et al. Real-life assessment of aripiprazole monthly (Abilify Maintena) in schizophrenia: a Canadian naturalistic non-interventional prospective cohort study. BMC Psychiatry 2019;19:114.

21. Peters-Strickland T, Baker RA, McQuade RD, Jin N, Eramo A, Perry $\mathrm{P}$, et al. Aripiprazole once-monthly $400 \mathrm{mg}$ for long-term maintenance treatment of schizophrenia: a 52-week open-label study. NPJ Schizophr 2015;1:15039.

22. Schreiner A, Caspi A, Bergmans P, Cherubin P, Keim S, Lara E, et al. Switching from oral atypical antipsychotic monotherapy to paliperidone palmitate once-monthly in non-acute patients with schizophrenia: a prospective, open-label, interventional study. Psychopharmacology (Berl) 2017;234:3-13.

23. Fleischhacker WW, Uchida H. Critical review of antipsychotic polypharmacy in the treatment of schizophrenia. Int I Neuropsychopharmacol 2014;17:1083-1093.

24. Nielsen J, le Quach P, Emborg C, Foldager L, Correll CU. 10-year trends in the treatment and outcomes of patients with first-episode schizophrenia. Acta Psychiatr Scand 2010;122: 356-366.

25. Gilmer TP, Dolder CR, Folsom DP, Mastin W, Jeste DV. Antipsychotic polypharmacy trends among Medicaid beneficiaries with schizophrenia in San Diego County, 1999-2004. Psychiatr Serv 2007;58:1007-1010.

26. Pae CU, Han C, Bahk WM, Lee SJ, Patkar AA, Masand PS. Effectiveness and tolerability of switching to aripiprazole once monthly from antipsychotic polypharmacy and/or other long acting injectable antipsychotics for patients with schizophrenia in routine practice: a retrospective, observation study. Clin Psychopharmacol Neurosci 2020;18:153-158.

27. Köhler O, Horsdal HT, Baandrup L, Mors O, Gasse C. Association between Global Assessment of Functioning scores and indicators of functioning, severity, and prognosis in first-time schizophrenia. Clin Epidemiol 2016;8:323-332.

28. Correll CU, Kishimoto T, Nielsen J, Kane JM. Quantifying clinical relevance in the treatment of schizophrenia. Clin Ther 2011;33:B16-B39.

29. Christensen AF, Poulsen J, Nielsen CT, Bork B, Christensen A, Christensen M. Patients with schizophrenia treated with aripiprazole, a multicentre naturalistic study. Acta Psychiatr Scand 2006; 113:148-153.

30. Suzuki T, Uchida H, Sakurai H, Ishizuki T, Tsunoda K, Takeuchi $\mathrm{H}$, et al. Relationships between global assessment of functioning and other rating scales in clinical trials for schizophrenia. Psychiatry Res 2015;227:265-269.

31. Samara MT, Engel RR, Millier A, Kandenwein J, Toumi M, Leucht $\mathrm{S}$. Equipercentile linking of scales measuring functioning and symptoms: examining the GAF, SOFAS, CGI-S, and PANSS. Eur Neuropsychopharmacol 2014;24:1767-1772.

32. Chue P, Malla A, Bouchard RH, Lessard S, Ganesan S, Stip E, et al.; SPECTRUM XR Study Group. The Iong-term clinical benefit and effectiveness of switching to once-daily quetiapine extended release in patients with schizophrenia. Curr Med Res Opin 2013;29:227-239.

33. Han C, Wang SM, Seo HJ, Lee BC, Jeon HJ, Kim W, et al. 
Aripiprazole augmentation, antidepressant combination or switching therapy in patients with major depressive disorder who are partial- or non-responsive to current antidepressants: a multi-center, naturalistic study. J Psychiatr Res 2014;49: 75-82.

34. Woo YS, Bahk WM, Pae CU, Jeong JH, Koo BH, Jon DI, et al. Observational study to evaluate the clinical benefit of lamotrigine add-on therapy in bipolar patients in a naturalistic treatment setting. Asia Pac Psychiatry 2014;6:334-341.

35. Guy W. ECDEU assessment manual for psychopharmacology. Rockville:U.S. Dept. of Health, Education, and Welfare, Public Health Service, Alcohol, Drug Abuse, and Mental Health Administration, National Institute of Mental Health, Psychopharmacology Research Branch, Division of Extramural Research Programs; 1976.

36. Konsztowicz S, Schmitz N, Lepage M. Dimensions of insight in schizophrenia: Exploratory factor analysis of items from multiple self- and interviewer-rated measures of insight. Schizophr Res 2018;199:319-325.

37. Siu CO, Harvey PD, Agid O, Waye M, Brambilla C, Choi WK, et al. Insight and subjective measures of quality of life in chronic schizophrenia. Schizophr Res Cogn 2015;2:127-132.

38. Chue P. The relationship between patient satisfaction and treatment outcomes in schizophrenia. I Psychopharmacol 2006;20(6 Suppl):38-56.

39. Marinis TD, Saleem PT, Glue P, Arnoldussen WJ, Teijeiro R, Lex A, et al. Switching to long-acting injectable risperidone is beneficial with regard to clinical outcomes, regardless of previous conventional medication in patients with schizophrenia. Pharmacopsychiatry 2007;40:257-263.

40. Potkin SG, Loze JY, Forray C, Baker RA, Sapin C, PetersStrickland T, et al. Multidimensional assessment of functional outcomes in schizophrenia: results from qualify, a head-tohead trial of aripiprazole once-monthly and paliperidone palmitate. Int J Neuropsychopharmacol 2017;20:40-49.

41. Kane JM, Sanchez R, Baker RA, Eramo A, Peters-Strickland T, Perry PP, et al. Patient-centered outcomes with aripiprazole once-monthly for maintenance treatment in patients with schizophrenia: results from two multicenter, randomized, double-blind studies. Clin Schizophr Relat Psychoses 2015;9: 79-87.

42. Kamei H, Homma Y, Takeuchi I, Hajitsu G, Tozawa K, Hatano $\mathrm{M}$, et al. Acceptance of the deltoid muscle injection of aripiprazole long-acting injectable in the patients with schizophrenia. Clin Psychopharmacol Neurosci 2020;18:49-57. 